\title{
COMENTARIO AL PALEOLÍTICO IBÉRICO: CONTINUIDAD, ETAPAS Y PERDURACIONES DEL PROCESO TECNOCULTURAL
}

\author{
A COMMENT ON IBERIAN PALEOLITHIC: CONTINUITY, STAGES AND \\ PERDURATIONS OF THE TECHNOCULTURAL PROCESS
}

\author{
por \\ ENRIQUE VALLESPÍ PÉREZ
}

\begin{abstract}
RESUMEN A escala general de la Península Ibérica, el Paleolítico Inferior aparece, en su aspecto tecnocultural, como un proceso de continuidad desde sus testimonios iniciales y el Achelense Ibérico Antiguo y su desarrollo en Achelense Pleno, con perduraciones en un Paleolítico Medio dual, de complejos musterienses y postachelenses, con préstamos recíprocos y unas perduraciones regionales terminales, en el Paleolítico Superior Antiguo.
\end{abstract}

\begin{abstract}
At a general Iberian Peninsula scale, Lower Paleolithic appears, from a technocultural point of view, as a continuous process that begins with the initial evidences and Early Iberian Acheulean. It develops towards the Full Acheulean to go on with a dual Middle Paleolithic represented by musterien complexes and postachelense perdurations, lastly presenting borrowings and terminal regional perdurations during the Early Upper Paleolithic.
\end{abstract}

Los registros actualmente disponibles del Paleolítico Inferior de la Península Ibérica aparecen irregularmente distribuidos, concentrados sustancialmente, en su doble alcance espacio-temporal, a lo ancho de la vertiente atlántica, y en menor escala en la mediterránea, muy escasos y en general más tardíos y, en parte, con aspecto comparativamente diferenciado, no sabemos si por desigualdad de investigaciones específicas o como prefiguración de sendas realidades del pasado paleolítico inferior de ambos grandes territorios ibéricos, $\mathrm{o}$, como es más que probable, por ambas circunstancias a la vez, cuya valoración no podemos sopesar. Pero esto es lo que hay y nos obliga a afrontar con provisionalidad toda ordenación de conjunto e intento de síntesis, lo que, por lo demás, resulta inherente a todo quehacer arqueológico.

Es, en efecto, en la vertiente atlántica, con concentraciones regionales de yacimientos estratificados en las grandes depresiones fluviales de la Región Central y, en un caso, en formación cárstica limítrofe, donde radican las secuencias estratigráficas claves del yacimiento de Atapuerca, de las depresiones fluviales de la Meseta Central (Submeseta Norte y Depresión española del Tajo, conjuntamente) y la del Bajo Guadalquivir, secuencias que por sus continuidades de gran alcance cronológico y el distanciamiento geográfico entre ellas (desde Atapuerca, en la encrucijada de las estribaciones cantábricas, 
corredor vasco-francés y divisorias de aguas de Ebro y Duero, a la secuencias fluviales de ambas submesetas, hasta el extremo sur de España) permiten estructurar, en planteamiento de momento teórico pero ya sobre sólidas bases estratigráficas reales, una secuencia regional estándar de gran alcance para la vertebración del Paleolítico Inferior de la Península Ibérica. A esta secuencia general, estructurada en lo que constituye el área nuclear del Achelense Ibérico, al menos tal como aparece en el estado actual de nuestros conocimientos, se correlacionan, avanzando el proceso, las secuencias regionales del Pleistoceno Medio y comienzos del Superior de la Periferia Septentrional, del Bajo Miño y de la franja cantábrica, así como el mundo inferopaleolítico de la Periferia Occidental, lo que permite prefigurar el proceso del Paleolítico Inferior de toda la vertiente atlántica de la Península, en su continuidad general y la matización de etapas achelense y de perduraciones postachelenses.

En contraste con este modelo de homogeneidad achelense, en la vertiente mediterránea, aunque debamos relativizar de momento la valoración cultural de esta división geográfica, que puede tener no obstante algún sentido, junto a la escasez de yacimientos y localizaciones controladas, se evidencia la diferenciación de sus registros en dos series tecnoculturales geográficamente separadas: a) de focos locales achelenses en su franja septentrional, en la Depresión alta y media-alta del Ebro y en el Noreste catalán, más significativamente, en relación con las series interiores colindantes y el mundo ultrapirenaico, con probabilidad, respectivamente; y b) un foco inferopaleolítico netamente diferenciado, al menos por sus registros disponibles, en la zona central de la Periferia Levantina, con aspecto no achelense y alcance impreciso, pero que parecen prefigurar un proceso diferencial, no bien definido todavía, del modelo de continuidad achelense avanzado y postachelense inmediato, generalizado en la Península.

Se expone seguidamente un intento de sistematización de cuanto sugieren estas reflexiones preliminares.

\section{HACIA UNA SECUENCIA GENERAL DEL ACHELENSE IBÉRICO}

La hipótesis de la estructuración, con los paralelismos secuenciales de Atapuerca y de las depresiones fluviales del Tajo y del Bajo Guadalquivir, de una secuencia general fiable del Paleolítico Inferior de la Región Céntral y del Sur, con proyección a la periferia occidental y septentrional de la Península, aparece claramente prefigurada en estos momentos, con el conocimiento actual de las secuencias regionales sustentantes y, en mi opinión, evidencia la continuidad del proceso paleolítico inferior de toda la vertiente atlántica ibérica, con alcance peninsular.

Desde estos puntos de vista, condicionados a la verificación de la hipótesis, con toda su complejidad y con las consecuencias interpretativas que con el tiempo resulten, la discusión de la continuidad secuencial del proceso puede ahora fundamentarse, en sus grandes líneas, con la comprobación del ensamblaje cronoestratigráfico de las tres citadas secuencias regionales clave, en sus nexos entre los tramos cruciales del proceso: $1^{\circ}$, entre los testimonios finales del Pleistoceno Inferior y los registros con industrias de diagnóstico achelense de comienzos del Pleistoceno Medio; $2^{\circ}$, en la continuidad del proceso estratigráfico achelense durante todo el Pleistoceno Medio; y $3^{\circ}$, en la perduración, sin solución de continuidad, de complejos achelenses en el Pleistoceno Superior Antiguo.

$1^{\circ}$. La correlación de los registros paleolíticos del Pleistoceno Inferior de Atapuerca (Gran Dolina TD4, TD5, TD6) y de la Depresión de Guadix-Baza (Fuente Nueva 3 y Barranco del Lobo 5, en el área de Orce) con los de comienzos del Pleistoceno Medio resulta evidente en ambas series secuenciales (consolidada en Atapuerca TD6 y TD7 y siguientes, y en Cúllar-Baza 1 y algún nexo pendiente en estudio, en Guadix-Baza), que a su vez se corresponden geocronológicamente con los registros iniciales de las secuencias fluviales del Sur y Centro de la Península, como se comprueba en el Bajo Guadalquivir, cuya T5, primer nivel con 
polaridad magnética normal, que inicia su Complejo de Terrazas Altas y presentan los primeros registros paleolíticos en conexión estratigráfica (Cerro Higoso I) de la secuencia, debe relacionarse con TD7 o en su caso TD6 de Atapuerca, en unos paralelismos afianzados en la propia secuencia del Bajo Guadalquivir y que debe reforzarse con atribuciones de la Región Central, tan evidentes como la de San Bartolomé de las Abiertas, nivel $+60 / 65 \mathrm{~m}$. del Tajo en la zona de Talavera de la Reina.

$2^{\circ}$. Sin solución de continuidad con los registros iniciales anteriores, el proceso paleolítico del Pleistoceno Medio está plenamente comprobado en la secuencia del Bajo Guadalquivir, con industrias ininterrumpidas en los depósitos de todos sus niveles de terrazas: desde la referida T5, primera del Complejo de Terrazas Altas, con muestras tecnológicamente indeterminadas, y achelenses bien definidos en T6 y abundantes en T7, T8 y T9, del Pleistoceno Medio Antiguo, y asimismo abundantes en las Terrazas Medias, T10 y T11, del Pleistoceno Medio Reciente, y en T12, media-baja transicional, del IG1 Riss-Würm.

Esta comprobación de la continuidad de los registros paleolíticos en la secuencia del Bajo Guadalquivir debe considerarse como expresión regional de un fenómeno generalizado en las grandes depresiones fluviales del Centro y Sur de la Península, aunque en las secuencias del Duero y del Tajo la conexión entre los testimonios disponibles de las terrazas altas y los muy abundantes de las terrazas bajas esté aún pendiente de su aclaración definitiva, en un vacío, sin duda, más aparente que real.

$3^{\circ}$. La continuidad del proceso paleolítico inferior se prolonga finalmente en una perduración de registros achelenses en el Pleistoceno Superior Antiguo, claramente evidenciada en el complejo de terrazas bajas de la Depresión Inferior del Guadalquivir, en relación con sedimentos superiores de la referida terraza media-baja, T12, y en los depósitos de la terraza baja, T13, de dicho río, así como en el Guadalete, de la misma Depresión. Análogos ejemplos de perduración han sido señalados en formaciones fluviales de ambas Submesetas y en algunos focos septentrionales de la Península. Las conocidas evidencias achelenses en las bases de las secuencias en cuevas con estratigrafías musterienses y en complejos tecnotipológicos de esos mismos niveles, refuerzan además el interés y alcance, no bien valorado todavía, de este fenómeno de perduraciones achelenses en el Paleolítico Medio de nuestra Península, prolongado con epígonos no desdeñables en los tiempos del Paleolítico Superior Antiguo, conforme se analiza más adelante, en el panorama general del proceso'.

\section{ORDENACIÓN DEL PROCESO. PRIMERA ETAPA: TESTIMONIOS INICIALES Y ACHELENSE IBÉRICO ANTIGUO}

Los registros paleolíticos anteriores a los achelenses hasta ahora diagnosticados, con el distanciamiento geográfico de sus yacimientos de Atapuerca y Orce, evidencian cierta dispersión territorial a escala peninsular, en relación con la Región Central y la Periferia meridional, que constituyen el área nuclear del Achelense Ibérico Antiguo actualmente controlado, a cuyos complejos tecnoculturales deben, con toda probabilidad, asimilarse, a mi modo de ver, por su evidenciada continuidad secuencial y por el horizonte del proceso intercontinental en que se insertan.

1. La discusión de esta secuencia general que se propone puede orientarse cómodamente con los siguientes trabajos recientes, reunidos en AEQUA (1997): sobre Atapuerca, Aguirre (1997), con repertorio bibliográfico, del que interesa aquí especialmente las entregas en curso de Carbonell y otros (1995); sobre Orce, Martínez Navarro y otros (1997), así mismo con bibliografía hasta esa fecha; de la Región Central, la actualización de Santonja y Pérez González (1997); y de nuestro proyecto "Formaciones Cuaternarias y secuencia paleolítica del Bajo Guadalquivir", los informes de Díaz del Olmo y Baena (1997), en la mencionada obra colectiva. 
La antigüedad de estos achelenses ibéricos, relativizado su calificativo de antiguo al ámbito peninsular y occidental, y la continuidad de su proceso son patentes por su significación cronoestratigráfica en la secuencia regional estándar sustentante. Su conocimiento, no obstante, queda de momento relegado a la publicación adecuada de los registros estratigráficos en estudio de Atapuerca y del Bajo Guadalquivir y a la verificación de los controles secuenciales mantenidos en su atribución del Duero y Tajo, principalmente, así como de la ampliación de la muestra estratigráfica disponible de la Cuesta de Baza, significativa como indicación de la continuidad local de los registros más antiguos de Orce. Sobre estas perspectivas inmediatas de conocimiento del Achelense Ibérico Antiguo puedo destacar la solidez argumental que presenta la valoración del Pleistoceno Medio Antiguo del Bajo Guadalquivir, con registros paleolíticos en la totalidad de los niveles de su Complejo de Terrazas Altas, con achelense bien caracterizado, con muestra de momento reducida en T6 y muy abundante en T7, T8 y T9, este último nivel datado en no menos de 300.000 B.P., según las seriaciones absolutas obtenidas ${ }^{2}$.

\section{SEGUNDA ETAPA: PLENO ACHELENSE IBÉRICO Y FACIES NO ACHELENSE DEL LEVANTE ESPAÑOL}

La segunda etapa del proceso, en el Pleistoceno Medio Reciente, desde alrededor de 300.000 B.P., se nos presenta caracterizada, en el estado actual de los conocimientos, por la generalización del achelense a toda la Vertiente Atlántica y a la Depresión del Ebro y del Nordeste, y paralelamente, por la prefiguración en el área mediterránea, en su zona levantina principalmente, de un complejo tecnocultural no achelense, no bien definido todavía. Ambos procesos tecnoculturales penetrarán, sin solución de continuidad, en los tiempos del Pleistoceno Superior Antiguo, dentro ya del Paleolítico Medio.

De este Achelense Pleno, constituido por la mayoría de los yacimientos achelenses de la Península conocidos, interesa aquí recordar el mapa de su distribución geográfica, que integra a todas las regiones de la vertiente atlántica y presenta: un aumento considerable de yacimientos en la gran área del Achelense Antiguo; su extensión generalizada por las periferias Occidental y Septentrional, con indicios anteriores a tener en cuenta, lo mismo que en el Nordeste de la Península; y la aparición de un foco en la cuenca mediaalta y valle medio del Ebro. De este modo, constituida la Iberia del Pleno Achelense en la vertiente atlántica sustancialmente, su delimitación en los territorios de vertiente mediterránea queda actualmente prefigurada, aparte del foco achelense de Nordeste catalán (en relación con el mundo pirenaico, pero aislado de momento del contexto achelense peninsular), por: la proyección del Achelense Pleno en el Ebro medio, desde la conjunción de la Submeseta Norte y la Periferia Septentrional con su depresión fluvial; el foco albaceteño de los achelenses de La Mancha, y en relación con ello, los registros achelenses del interior de la región murciana, enlazando así con el área achelense de la Alta Andalucía.

Desde esta perspectiva de la distribución geográfica de los registros hasta ahora disponibles, la periferia mediterránea, al Este y Sudeste de la delimitación del Achelense Pleno trazada, aparece de momento sin controles de registros asimilables, mientras que, por el contrario, el único yacimiento en excavación y estudio, la cueva de Bolomor, en la comarca de Gandía, presenta dos niveles de un paleolítico inferior tecnotipológicamente no achelense, plenamente coetáneos del proceso del Pleno Achelense peninsular

2. Como referencias orientadoras de esta primera etapa creo suficientes las indicadas en la precedente nota. Sobre el período inicial de Atapuerca me ha servido también mi conocimiento de la tesis doctoral inédita de X.P. Rodríguez Álvarez (1997); para la depresión de Guadix-Baza son clarificadores los Informes de la Comisión Asesora Internacional, en la que participo, emitidos en 1997, 1998 y 2000, inéditos en la Consejería de Cultura de la Junta de Andalucía. Con respecto a la vertebración del Achelense Ibérico Antiguo, insisto especialmente en el interés del argumento secuencial del Bajo Guadalquivir, con registros paleolíticos cumplidamente documentados en sendas tesis doctorales inéditas, de J.J. Fernández Caro (1998) y de J.A. Caro Gómez (1999). 
y acompañado, al parecer, por indicios similares en su contexto local. Además, otro yacimiento, del interior turolense en la Cordillera Ibérica, la Cuesta de la Bajada, también en curso de excavación, ofrece un registro que pudiera tal vez asimilarse a momentos tardíos de dicho proceso, lo que hace pensar en la existencia de una facies regional, en la vertiente mediterránea levantina, con su foco nuclear en la misma periferia litoral, de un Paleolítico Inferior no achelense, en proceso paralelo al Pleno Achelense en todo el amplio resto de la Península ${ }^{3}$.

\section{TERCERA ETAPA: POSTACHELENSE DEL PALEOLÍTICO MEDIO}

La continuidad tecnocultural del Paleolítico Inferior culmina en la Península Ibérica con un fenómeno de perduraciones achelenses, de extensión y alcance aún sin estudiar, pero que aparece suficientemente prefigurado en las formaciones de las grandes depresiones fluviales del Centro y Sur de la Península, así como en las Periferias Occidental y Septentrional y en el Nordeste catalán, evidenciando de este modo su amplia generalización en las grandes extensiones territoriales de la Iberia del Achelense Pleno. Curiosamente también, en la facies no achelense de litoral levantino, en la mencionada estratigrafía de Bolomor, los registros de sus niveles superiores son asimismo una continuidad en su proceso secuencial.

Ambas líneas tecnoculturales constituyen, por lo tanto, sendas continuidades en el Paleolítico Medio de sus anteriores procesos diferenciados, cuyas divergencias aumentan su contraste en la nueva etapa: mientras las perduraciones achelenses son una mera continuidad tecnotipológicamente estacionaria, postachelenses como simple continuismo, en el proceso de la facies no achelense, al menos en la cueva citada, se trata de una continuidad dinámica, evolutiva, transformadora de unos complejos sobre lasca en lo que se ha calificado, y lo repito aquí en su sentido indicativo, de "premusteriense o musteriense", cuestión ésta de la génesis de los musterienses, compleja y de mayor alcance, al margen, como es obvio, de este comentario.

En este proceso de continuidad puede pensarse en distinguir, al menos teóricamente, un fase transicional del Paleolítico Inferior avanzado y final al Paleolítico Medio, que estaría definida fundamentalmente por las perduraciones achelenses en los territorios de la Iberia del Achelense Pleno, ya que la concreta referencia del área no achelense a un único yacimiento no permite, por ahora, una interpretación fiable de las raíces del proceso, no descartable desde lo achelense, y en este sentido cabría asimismo la posibilidad de pensar en alguna transformación análoga en lugares de la Iberia Achelense hacia lo premusteriense o musteriense tempranos. Quede al menos como pensamientos colaterales del propósito de estas notas.

La nueva situación resultante, en la que cabe considerar segunda fase, plenamente del Paleolítico Medio, presenta una redistribución de los registros paleolíticos, casi la inversión territorial, a escala peninsular,

3. Casi todos los yacimientos achelenses excavados en la Península Ibérica pertenecen a esta Segunda Etapa del proceso, con la consiguiente acumulación bibliográfica, de la que, abreviando, en relación con el presente texto, por su alcance estratigráfico en la secuencia general ibérica, interesa recordar:

Sobre Centro y Sur peninsulares: para la Meseta, Santonja y Pérez González (1997), cuya revisión sitúa a Pinedo, de especial significado cronológico en la bibliografía precedente, y a Ambrona y Torralba en el Pleistoceno Medio Reciente; sobre el Bajo Guadalquivir, con excelentes perspectivas de excavación en prometedores yacimientos estratificados en la secuencia, son fundamentalmente las tesis doctorales inéditas de J.J. Fernández Caro (1998) y J.A. Caro Gómez (1999)

De la Periferia Septentrional interesa primordialmente la secuencia regional fundamentada por Rodríguez Asensio, básicamente en Cabo Busto, junto a otros yacimientos asturianos excavados (Rodríguez Asensio y Noval Fonseca, 1998) y también en su extensión cantábrica (R. Montes Barquín, 1999).

En el Noroeste, la secuencia planteada en el Bajo Miño abre nuevas perspectivas a lo conocido hasta ahora en las Gándaras de Budiño (Cano y otros, 1997).

Sobre Bolomor, Fernández Peris y otros (1997). 
del panorama anterior, con los complejos musterienses, grosso modo, densificados en las periferias peninsulares y en las regiones montañosas interiores, y dejando las grandes depresiones fluviales del centro y Sur, es decir, las viejas áreas del Achelense Antiguo, con postachelenses residuales, que alcanzarían, como veremos luego, al Pleistoceno Superior Antiguo.

En este panorama peninsular, la conjunción de ambos mundos ambientales, de ocupación de territorios con cavidades en zonas montañosas y de campo abierto en depresiones fluviales, desde los tiempos finales del Paleolítico Inferior a los postachelenses residuales y musterienses del Paleolítico medio, originaría diversas interacciones tecnoculturales, tales como ciertos impactos recíprocos y la presencia de registros achelenses basales en cuevas con secuencias musterienses.

La distribución de testimonios conocidos de ésta última manifestación, de estratigrafías musterienses superpuestas a depósitos basales con registros tecnotipológicamene achelenses, demuestran su extensión en la España caliza, en Cantabria con la clásica Cueva del Castillo y las de Horá y la casi inédita del Ángel, en la Alta Andalucía, donde La Solana del Zamborino puede tal vez corresponder a la modalidad en campo abierto de estas superposiciones musterienses sobre base con registros achelenses.

Como manifestación del mismo fenómeno, los intercambios tecnotipológicos recíprocos entre los postachelenses y los complejos musterienses presentan evidencias muy significativas, en su doble sentido:

a) impactos de lo postachelense en estratigrafías musterienses, que aparecen singularmente en la Prehistoria Septentrional, desde Asturias al País Vasco, y por reducción a lo meramente musteriense han sido considerados en la historiografía como "facies vasconiense": al clásico registro de Castillo, junto a otras evidencias menores, hay que añadir el descubrimiento reciente de La Viña, con un significativo impacto achelense en el estrato basal musteriense, de contenido tecnotipológico excepcional y subyacente del Auriñaciense Antiguo de la secuencia, lo que prueba la duración postachelense hasta un musteriense muy avanzado.

y b) impactos musterienses en el postachelense, cuyas evidencias parecen distribuidas por los amplios espacios regionales de perduraciones de vieja tradición achelense, en las terrazas bajas y formaciones correlacionadas, de arroyos y coluviones, de las grandes depresiones fluviales de la Región Central, Periferia Occidental y Baja Andalucía, así como en la Periferia Septentrional y en el Nordeste catalán. Se trata en todos los casos de complejos tecnotipológicos mixtificados con impactos musterienses, manifestación de un Paleolítico Medio Ibérico no clásico ${ }^{4}$.

4. Sobre esta cuestión de las perduraciones achelenses en el Paleolítico Medio, con viejas referencias del foco clásico del Manzanares y acumulación de nuevas evidencias posteriores, puede verse mi trabajo "Paleolítico Medio indeterminado, de aspecto postachelense, en ámbitos fluviales del Sur y entro de la Península Ibérica" (Vallespí, 1989), donde remito las referencias bibliográficas omitidas en el presente texto. La referencia a Bomor en J. Fernández Paris y otros (1997).

La valoración que presento de evidencias postachelenses en el musteriense de La Viña y el conocimiento de las mismas lo debo directamente al director de su excavación, Javier Fortea.

En relación con las experiencias de perduraciones achelenses regionales, debo recordar: que el "epiachelense" aducido por Santonja (1984) en su memoria de La Maya, es relacionable con dicho fenómeno; de la Periferia Septentrional es también referencia obligada la postura de Rodríguez Asensio sobre la perduración achelense en el Paleolítico Medio de Asturias, mantenida desde 1983 reiteradamente (cfs. Rodríguez Asensio y Noval Fonseca, 1998); de la Periferia Occidental, sin un conocimiento directo, que lamento, me limito a expresar mi impresión de síntesis en el sentido de su correspondencia con el mundo de las mixtificaciones postachelenses que planteo; en el Nordeste catalán, el argumento de dichas perduraciones parece asimismo evidente.

Es la estructuración de la secuencia del Bajo Guadalquivir, sistemáticamente trabajada desde 1982, la que nos ha evidenciado el sentido secuencial, dinámico, del proceso achelense hasta su integración en el Paleolítico Medio de sus niveles bajos de terrazas, comprobado también el río Guadalete, de la misma Depresión Inferior del Guadalquivir por F. Giles y colaboradores. 


\section{EPÍGONO: POSTACHELENSES TERMINALES, EN EL PALEOLÍTICOSUPERIOR ANTIGUO}

Llegados a este punto, se impone una reflexión sobre los epígonos del proceso hasta aquí planteado, cuya argumentación se apoya radicalmente en la idea de la continuidad: continuidad de los testimonios iniciales y el Achelense Antiguo y su desarrollo, sin solución de continuidad, en Achelense Pleno y, en el Paleolítico Medio, de sus prolongaciones postachelenses, en paralelo y con mutuas conexiones con los complejos musterienses avanzados.

Desde esta perspectiva de un Paleolítico Medio tecnoculturalmente dual, de complejos musterienses y perduraciones postachelenses con préstamos recíprocos, es del todo coherente preguntarse hasta cuándo, en los tiempos transicionales y adentrado el Paleolítico Superior Antiguo, pueden rastrearse evidencias de sus últimos registros tangibles, tecnotipológicos en los que se sustenta la hipótesis, que fijen el final del proceso de la continuidad postachelense.

En este sentido, además de las pruebas que pueden aportarse de los ámbitos musterienses clásicos, con impactos achelenses tan significativos como los aducidos de las Periferias Septentrional y Meridional y otros que pueden añadirse, resulta especialmente reveladora la generalización en la Periferia Occidental de complejos del Paleolítico Medio considerados del Musteriense clásico en su aceptación tradicional al uso, pero que deben corresponder a los complejos mixtificados, postachelenses con impacto musteriense, aquí propuestos, y que presentan dataciones muy tardías, en torno al 25.000 B.P. Son también postachelenses con préstamos musterienses los complejos de la Baja Andalucía y lo mismo cabe decir para las series semejantes de la Región Central. Con referencia a la misma Periferia Meridional, pero de la Alta Andalucía, conviene recordar, por su relación en este asunto, el alcance igualmente tardío y coincidente con dicha cronología, de las dataciones del musteriense clásico de la cueva de La Carigüela y del abrigo de Zafarraya.

De la valoración de todos estos indicios ahora disponibles, cabe deducir que en el mundo de los postachelenses regionales de las depresiones fluviales de ambas Submesetas, la Periferia Occidental y la Baja Andalucía, principalmente, perduraron por lo tanto sus complejos mixtificados hasta, al menos, los tiempos de los auriñacienses avanzados de la secuencia clásica, reducida ésta, a escala peninsular, con su proceso secuencial que nos atañe, Chatelperronense en su caso, auriñaciense y gravetiense, a los territorios ibéricos periféricos y montañosos anteriormente ocupados por los complejos musterienses.

De confirmarse esta hipótesis, estaríamos, por lo tanto, ante una nueva perspectiva del Paleolítico Superior Antiguo en la Península Ibérica, distinta de la tradicionalmente considerada ${ }^{5}$.

5. En mi atención a los procesos poblacionales de ámbitos regionales, he tenido ocasión de exponer mi reflexión sobre la existencia de grandes espacios vacíos de registros paleolíticos desde el final de los musterienses hasta el período solutrense, en amplios territorios e incluso hasta mucho después en territorios más restringidos, de nuestra Península, de cuya preocupación he dejado testimonio en varios trabajos, sobre la Región Castellano-Manchega y el Alto y Bajo Guadalquivir. Tales ideas están en la base del texto, en el que ahora pretendo fijar el final del legado tecnotipológico de las perduraciones postachelenses en tiempos del Paleolítico Superior Antiguo.

Pienso que, por la amplitud espacial y temporal de estos vacíos de registros paleolíticos y aunque se relativice el alcance real del fenómeno en su actual apariencia, no debe seguir soslayándose en la interpretación del panorama paleolítico ibérico. En el texto, mi alusión a las series musterienses tardías de la periferia occidental las tomo de la sistematización de Ignacio Barandiarán (1998). 


\section{BIBLIOGRAFÍA}

Advertencia bibliográfica: Téngase en cuenta que la lista que acompaña se limita a una ficha por autor/es, pero remite implícitamente a los trabajos implicados en el tema, de los mismos y de otros autores.

AAVV (1997): J. Rodríguez Vidal (ed.), Cuaternario Ibérico. AEQUA. Huelva.

AGUIRRE, E. (1997): “Atapuerca y Cuaternario. Correlaciones, novedades e interrogantes”, en J. Rodríguez Vidal (ed.) Cuaternario Ibérico, Huelva: 213-223.

BARANDIARÁN, I. (1999): “El Paleolítico y el Mesolítico" Prehistoria de la Península lbérica. Ed. Ariel. Barcelona: 1-120.

CANO, J.A.; AGUIRRE, E.; GILES, F.; GRACIA, F.J.; SANTIAGO, A.; MATA, E.; GUTIÉRREZ, J.M.; DÍAZ DEL OLMO, F.; BAENA, R. y BORJA, F. (1997): "Evolución del Pleistoceno en la cuenca baja del Miño, sector La Guardia-Tuy. Secuencia de los primeros poblamientos humanos y registro arqueológico", en J. Rodríguez Vidal (ed.) Cuaternario Ibérico, Huelva: 201-212.

CARBONELL, E.; GIRALT, S.; MÁRQUEZ, B.; MARTÍN, A.; MOSQUERA, M.; OLLÉ, A.; RODRÍGUEZ, X.P.; SALA, R.; VAQUERO, M.; VERGES, J.M. y ZARAGOZA, J. (1995): "El conjunto lito-técnico de la Sierra de Atapuerca en el marco del Pleistoceno Medio europeo", en J.M. Bermúdez de Castro, J.L. Arsuaga y E. Carbonell, Evolución humana en Europa y los yacimientos de Atapuerca. Valladolid.

CARO GÓMEZ, J.A. (1999): Yacimientos e industrias achelenses en las terrazas fluviales de la depresión del Bajo Guadalquivir (Andalucía, España). Secuencia estratigráfica, caracterización tecnocultural y cronología, Tesis doctoral (inédita). Universidad de Sevilla.

DÍAZ DEL OLMO, F. y BAENA, R. (1997): “Interpretación de la secuencia general del Guadalquivir (Valle Medio y Bajo): terrazas fluviales y Paleolítico”, en J. Rodríguez Vidal (ed.) Cuaternario Ibérico, Huelva: 273-282.

FERNÁNDEZCARO, J.J. (1998): Las industrias líticas paleolíticas del Bajo Guadalquivir: Río Corbones. Tesis doctoral (inédita). Universidad de Córdoba.

FERNÁNDEZ PERIS, J.; GUILLEM, P.y MARTÍNEZ VALLE, R. (1997): Cova de Bolomor. Los primeros habitantes de las tierras valencianas. Museo de Prehistoria. Valencia.

FORTEA PÉREZ, J. (1999): “Abrigo de La Viña. Informe y primera valoración de las campañas de 1995 a 1998”, en Excavaciones Arqueológicas en Asturias 1995-98.

MARTÍNEZ, B.; PALMQVIST, P.; ARRIBAS, A.; TURQ, A.; AGUSTÍ, J. y OMS, O. (1997): "Síntesis de las investigaciones paleontológicas y arqueológicas en el Plio-Pleistoceno de la región de Orce", en J. Rodríguez Vidal (ed.) Cuaternario Ibérico, Huelva: 261-272.

MONTES BARQUÍN, R. (1998): Los complejos industriales del Paleolítico Inferior en la Región Cantábrica, Tesis doctoral (inédita). Universidad de Cantabria.

RODRÍGUEZ ÁLVAREZ, X.P.(1997): Los sistemas técnicos de producción lítica en el Pleistoceno Inferior y Medio de la Península Ibérica: variabilidad tecnológica entre yacimientos del Noreste y de la Sierra de Atapuerca, Tesis doctoral (inédita). Universitat Rovira i Virgili. Tarragona.

RODRÍGUEZ ASENSIO, J.A. y NOVAL FONSECA, M.A. (1998): Gijón antes de Gijón. Breve aproximación a los primeros grupos predadores en la Prehistoria de Asturias. Ayuntamiento de Gijón.

SANTONJA, M. y PÉREZ GONZÁLEZ, A. (1997): "Los yacimientos achelenses en terrazas fluviales de la Meseta Central española", en J. Rodríguez Vidal (ed.) Cuaternario Ibérico, Huelva: 224-234.

VALLESPÍ PÉREZ, E. (1989): "Paleolítico Medio indeterminado, de aspecto postachelense, en ámbitos fluviales del Sur y Centro de la Península Ibérica" Veleia 6: 7-20. 